\title{
Forme Fruste of Septic Arthritis in a Patient with Ulcerative Colitis
}

\author{
Arup Choudhury ${ }^{1}$, Deepak Kumar ${ }^{2}$, Pankaj Gupta ${ }^{3}$, Aman Sharma ${ }^{4}$, Vishal Sharma ${ }^{5}$
}

\begin{abstract}
Musculoskeletal involvement is the most common extraintestinal manifestation of inflammatory bowel disease (IBD). Typically, peripheral joint involvement in IBD is classified into two types depending upon involvement of joint pattern. A part from IBD associated arthritis, patient of IBD is also at risk of developing non-IBD related arthritis. Patients with IBD are on immunosuppressive medication and are at risk of developing septic arthritis, which is a medical emergency and needs emergent drainage and antibiotic therapy. Here we report a case of ulcerative colitis in a woman who was on azathioprine, presenting with unilateral pain and swelling of knee joint where a diagnosis of septic arthritis was established. The case highlights difficulties in diagnosis especially in patients on immunosuppression.
\end{abstract}

Keywords: Arthritis, Inflammatory bowel disease, Septic arthritis, Ulcerative colitis.

Journal of Gastrointestinal Infections (2021): 10.5005/jp-journals-10068-3051

\section{INTRODUCTION}

Inflammatory bowel disease (IBD) could be associated with many extraintestinal manifestations of which musculoskeletal involvement is a common one., ${ }^{1,2}$ Joint involvement could be either peripheral arthritis or axial spondyloarthropathy. However, given the need for immunosuppression to achieve and maintain disease remission, these patients could be at a heightened risk of infections. ${ }^{3,4} \mathrm{It}$ is unclear if the manifestations and synovial fluid analysis are impacted by underlying drug treatment including immunosuppression. We report about a lady in remission of ulcerative colitis and receiving mesalamine and azathioprine, who presented with pain and swelling of right knee with equivocal findings on evaluation.

\section{Case Description}

A 48-year-old female presented with two-day history of fever and painful swelling of right knee. She was suffering from ulcerative colitis since the last 21 years without any extraintestinal manifestation. According to the Montreal classification, the extent of ulcerative colitis was E3, i.e., extensive disease (beyond the splenic flexure). Her disease had relapsing and remitting course that necessitated multiple steroid courses with the last one given 3 years ago. She had done well on maintenance therapy with oral mesalamine $4.8 \mathrm{~g}$ and azathioprine $75 \mathrm{mg} /$ day since then. On examination, her right knee was swollen, tender with decreased active and passive movements of the right knee joint. Examination of rest of joints was normal. Right knee X-ray did not reveal any joint erosion (Fig. $1 \mathrm{~A}$ ) and ultrasound of right knee showed mild synovial thickening and effusion of the joint (Fig. 1B). Routine blood investigation showed hemoglobin of $10.8 \mathrm{~g} / \mathrm{L}$ with normal leukocyte count $\left(7400 / \mathrm{mm}^{3}\right)$. Serum uric acid level was $2.5 \mathrm{mg} / \mathrm{dL}$ and C-reactive protein was $135 \mathrm{mg} / \mathrm{L}$. Blood culture was sterile. Magnetic resonance imaging of knee showed joint effusion and synovial thickening and enhancement without any joint erosion (Fig. 1C). Routine stool works up was normal with fecal calprotectin of $75 \mu \mathrm{g} / \mathrm{g}$ of stool.

Ultrasound guided therapeutic and diagnostic aspiration of synovial fluid showed straw colored fluid with total count $11275 / \mathrm{mm}^{3}$, $89 \%$ polymorphonuclear cells, sugar $142 \mathrm{mg} / \mathrm{dL}$, and protein $3.8 \mathrm{~g} / \mathrm{dL}$.

\begin{abstract}
1,5Department of Gastroenterology, Postgraduate Institute of Medical Education and Research, Chandigarh, India

${ }^{2}$ Department of Orthopedics, Postgraduate Institute of Medical Education and Research, Chandigarh, India

${ }^{3}$ Department of Radiodiagnosis, Postgraduate Institute of Medical Education and Research, Chandigarh, India

${ }^{4}$ Department of Internal Medicine, Division of Clinical Immunology and Rheumatology, Postgraduate Institute of Medical Education and Research, Chandigarh, India
\end{abstract}

Corresponding Author: Vishal Sharma, Department of Gastroenterology, Postgraduate Institute of Medical Education and Research, Chandigarh, India, e-mail: docvishalsharma@gmail.com

How to cite this article: Choudhury A, Kumar D, Gupta P, et al. Forme Fruste of Septic Arthritis in a Patient with Ulcerative Colitis. J Gastrointest Infect 2021;11:42-43.

Source of support: Nil

Conflict of interest: None

Gram stain of the synovial fluid showed gram-negative bacilli but the culture did not grow any organism. Patient also underwent therapeutic aspiration of synovial fluid, of which $80 \mathrm{~mL}$ fluid was aspirated. In the wake of suspicion of septic arthritis, piperacillin and tazobactam were started and azathioprine was withheld which resulted in improvement in fever and resolution of pain, redness and swelling of the right knee joint. She was discharged after 4 days with oral antibiotic for 2 weeks, and after 4 weeks of follow-up, she did not have recurrence of joint symptoms and her stool frequency was two times without any blood in stool.

\section{Discussion}

This case highlights the clinical dilemma in diagnosing musculoskelteal system involvement in the patients with IBD. IBD related arthritis is typically divided into axial and peripheral arthritis. Arthritis in patients with IBD can be IBD associated or non IBD related. As per Oxford criteria, IBD-associated peripheral arthritis 

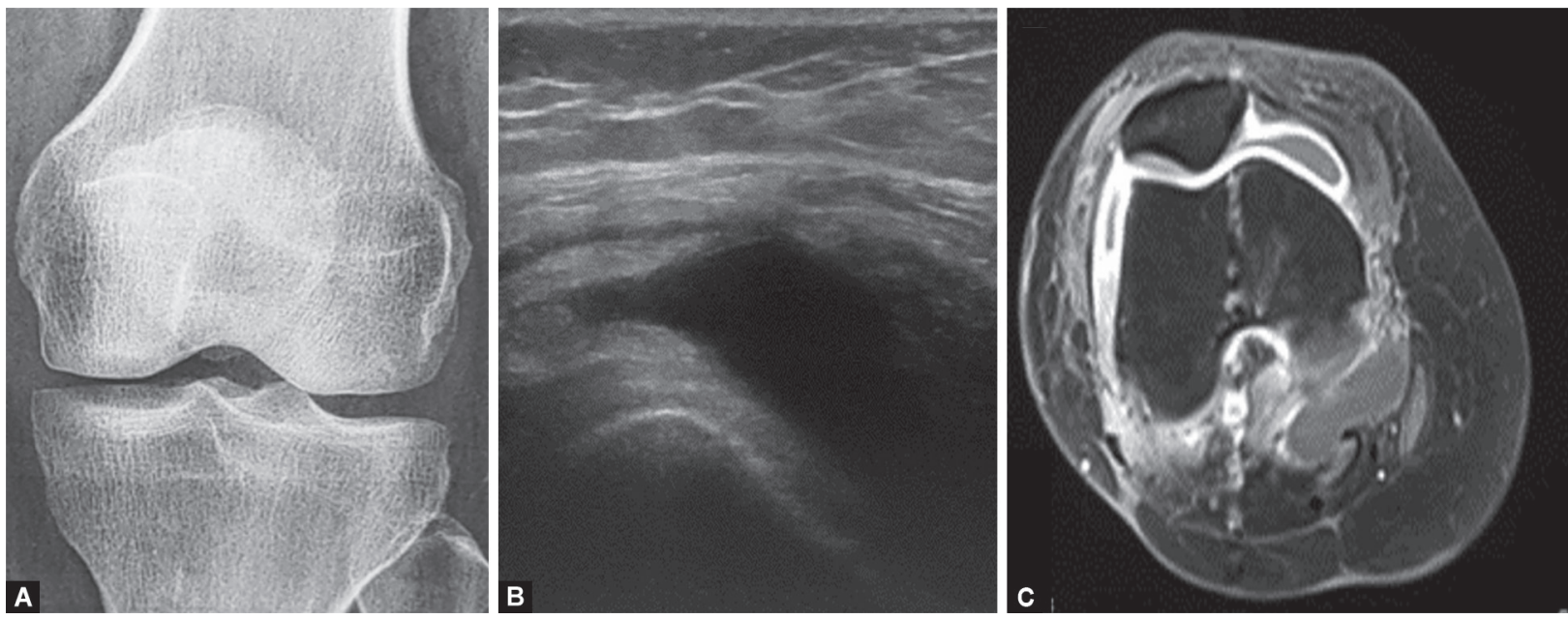

Figs 1 A to C: (A) Anteroposterior radiograph of right knee was unremarkable; (B) Longitudinal ultrasound image of the right knee shows joint effusion and mild synovial thickening; (C) Contrast-enhanced axial MR image of right knee shows joint effusion with synovial thickening and enhancement

are classified in to type I, which is mono or oligo arthritis ( $<5$ joints) asymmetrical predominantly involving the weight bearing large joints of lower limb, and type II, which is symmetrical polyarthritis predominantly involving the small joints of the hand. Type I arthritis, associated with activity of bowel disease, runs a course parallel to bowel disease but type II arthritis is not related to bowel disease and runs an independent course. ${ }^{2}$ While type I arthritis was a consideration in our patient, the lack of significant IBD activity and presence of monoarthritis with joint tenderness and redness argue against this possibility.

Differrential diagnosis of monoarthritis include septic arthritis, crystal induced arthropathy or trauma. Patients on immunosuppressive therapy have an increased risk of developing septic arthritis. ${ }^{3,4}$ Septic arthritis is a medical emergency and delay in medical therapy can cause permanent joint damge.

High leucocyte counts in synovial fluid (typically $>50,000 / \mathrm{mm}^{3}$ ) with $>90 \%$ polymorphs favor the diagnosis of septic arthritis. ${ }^{5}$ There are multiple reports which suggest that cell count could be lower in certain setting. However, the most definitive test to diagnose septic arthritis is synovial fluid culture. It is unclear if the patients who are on immune suppressant therapy mount an effective immune response and whether the leukocyte counts in synovial fluid may be lower than the typically described counts. These counts could also be lower in early disease course, partially treated septic arthritis, peripheral leukopenia, prosthetic joints, etc. In such a clinical situation a lower threshold of synovial fluid total leukocyte count should be used to avoid delay in treatment initiation. ${ }^{6-8}$ Our patients were on azathioprine and did not demonstrate peripheral leukocytosis, and this could be one reason for a lower cell count in the synovial fluid.

\section{Conclusion}

Bacterial infection of joint can progress rapidly causing cartilage damage, septicemia, and even death. So, a high index of clinical suspicion, early diagnosis, and treatment are essential to preserve the joint function and to have a good clinical outcome.

\section{References}

1. Jansen FM, Vavricka SR, den Broeder AA, et al. Clinical management of the most common extra-intestinal manifestations in patients with inflammatory bowel disease focused on the joints, skin and eyes. United European Gastroenterol J 2020;8(9):1031-1044. DOI: $10.1177 / 2050640620958902$.

2. Orchard TR, Wordsworth BP, Jewell DP. Peripheral arthropathies in inflammatory bowel disease: their articular distribution and natural history. Gut 1998;42(3):387-391. DOI: 10.1136/gut.42.3.387.

3. Chisari E, D'Mello D, Sherman MB, et al. Inflammatory bowel diseases increase the risk of periprosthetic joint infection. J Bone Joint Surg Am 2021. DOI: 10.2106/JBJS.20.01585.

4. Borman ZA, Côté-Daigneault J, Colombel JF. The risk for opportunistic infections in inflammatory bowel disease with biologics: an update. Expert Rev Gastroenterol Hepatol 2018;12(11):1101-1108. DOI: 10.1080/17474124.2018.1530983. PMID: 30277409.

5. Kaandorp CJ, Van Schaardenburg D, Krijnen P, et al. Risk factors for septic arthritis in patients with joint disease. A prospective study. Arthritis Rheum 1995;38(12):1819-1825. DOI: 10.1002/art.1780381215.

6. McCutchan HJ, Fisher RC. Synovial leukocytosis in infectious arthritis. Clin OrthopRelat Res 1990;(257):226-230. PMID: 2379361.

7. Ruzbarsky JJ, Gladnick BP, Dodwell E. Diagnosing septic arthritis in the synovial white cell count "Gray Zone". HSS J 2016;12(2):190-192. DOI: 10.1007/s11420-015-9480-6.

8. Horowitz DL, Katzap E, Horowitz S, et al. Approach to septic arthritis. Am Fam Physician 2011;84(6):653-660. PMID: 21916390. 